\title{
Posicionamiento, arquitectura e identidad de marca: un estudio correlacional en el sector calzado de Tungurahua.
}

Architecture and positioning, brand identity: a correlational study in the footwear sector of Tungurahua.

Luis Miguel Suárez Ramírez ${ }^{1}$, Juan Gabriel Saltos Cruz² , Carlos Javier Beltrán Avalos ${ }^{3}$

\begin{abstract}
.
DOI: https://doi.org/10.33262/cienciadigital.v2i2.75

The present investigation was oriented to the development and implementation of a brand management model that includes architecture, identity and positioning. Based on this model, it is expected to contribute with the business positioning in the footwear sector in the province of Tungurahua, considering that SMEs need to measure the value of their intangible assets, for this reason it was theoretically based and the positioning of the companies was diagnosed. different brands of the sector. The methodology was based on a categorical proposal that analyzed general theories from their sources and main authors. The classification of data was made through categories by abstract meanings, content and thematic unit. Based on the information gathered, it was possible to quantify the positioning of national companies according to consumer perception through the application of two instruments, the first was a structured survey applied to consumers of footwear in the province, the second was a formal structured interview with the owners of small and medium footwear companies in the province. The results obtained from the investigation revealed that the variables are valid in the measurement of architecture, brand identity and therefore the model is applicable as a tool for brand positioning in the mind of the consumer contributing to SMEs and the academic sector.
\end{abstract}

\footnotetext{
${ }^{1}$ Universidad Técnica de Ambato, F. Ciencias Administrativas Ambato, Ecuador, 1suarez2773@uta.edu.ec

${ }^{2}$ Universidad Técnica de Ambato, Universidad de la Habana,jg.saltos@uta.edu.ec

${ }^{3}$ Universidad Técnica de Ambato, F. Ciencias Administrativas Ambato, Ecuador, cj.beltran@uta.edu.ec
} 
Keywords: Brand Management, Brand Loyalty, Brand Awareness, Perceived Quality, Brand Partnerships

\section{Resumen.}

La presente investigación estuvo orientada a la elaboración e implementación de un modelo de gestión de marca que incluye la arquitectura, identidad y posicionamiento. A partir de este modelo se prevé que aporte con el posicionamiento empresarial en el sector del calzado en la provincia de Tungurahua, considerando que las PYMES necesitan medir el valor de sus activos intangibles, por esta razón se fundamentó teóricamente y se diagnosticó el posicionamiento de las diferentes marcas del sector. La metodología estuvo basada en una propuesta categorial que analizó teorías generales desde sus fuentes y principales autores. La clasificación de datos se efectuó mediante categorías por significados abstractos, de contenido y por unidad temática. En función de la información recolectada permitió calcular el posicionamiento de empresas nacionales según la percepción del consumidor mediante la aplicación de dos instrumentos, el primero fue una encuesta estructurada aplicada a los consumidores de calzado de la provincia, el segundo fue una entrevista formal estructurada a los propietarios de pequeñas y medianas empresas de calzado de la provincia.

Los resultados obtenidos de la investigación revelaron que las variables son válidas en la medición de arquitectura, identidad de marca y por consiguiente el modelo es aplicable como una herramienta para el posicionamiento de marca en la mente del consumidor aportando a las PYMES y al sector académico.

Palabras Claves: Gestión de marca, lealtad de marca, conciencia de marca, calidad percibida, asociaciones de marca

\section{Introducción.}

El calzado en el mercado mundial se encuentra encabezado por empresas pymes en la gran mayoría, en ciertos casos se hallan organizaciones de distintos tamaños, estas empresas en reducida cantidad son marcas internacionales que suministran mundialmente calzado de diferente modalidad de producción como son: producción trasnacional, producción nacional, maquila, etc. (BID \& INTAL, 2000), por esta razón, aparece la externalización como modalidad de producción que da la posibilidad de que pequeñas empresas se conviertan en proveedores de materia prima (Villegas \& Zapata, 2007). 
En la última década, en el Ecuador el sector calzado ha enfrentado grandes dificultades debido a la dolarización que generó la desaparición de empresas y devaluaciones, otro factor que afecto a la industria fue la importación de bienes a precios inferiores ofrecidos nacionalmente, lo que desembocó en muchos artesanos cambiar de sector debido a la competitividad del precio (Jácome, Naranjo, \& Mayo, 2010, pág. 7).

En la provincia de Tungurahua engloba el 44\% de calzado nacional, es decir, 44 de 100 pares son fabricados en la provincia, los cuales se clasifican en micro, pequeños y medianos productores, ubicados en diferentes cantones. (Comercio, 2016). En la provincia existe una organización conformada por 4500 productores nacionales de distinto tamaño dedicados a la elaboración y distribución de calzado llamada CALTU (Cámara de Calzado de Tungurahua).

Las Pymes localizadas en Tungurahua han tenido un crecimiento en su producción puesto que por la magnitud de su tamaño no es necesario tener procesos de gerencia, administración y operaciones, ya que son empresas pequeñas dirigidas por artesanos (Guajala, Mantilla, Mayorga, \& Moyolema, 2015). Por otro lado, el recurso económico es limitado, por esta razón las Pymes realizan créditos con proveedores o préstamos bancarios para cubrir su capital de operación (King \& Varela, 2012). Cabe mencionar al existir la dificultad para obtención de créditos obstaculiza su crecimiento ya que limita la importación de maquinaria, insumos y materia prima.

Por esta razón es importante analizar la influencia que tiene el modelo construido a partir de arquitectura, identidad y posicionamiento de marca en las empresas del sector de calzado de Tungurahua. A través de esta pregunta se pretende conocer la creación e implementación del modelo de gestión de marca repercute en el posicionamiento en la mente del consumidor e internamente en la empresa.

\section{Marco teórico.}

La marca monolítica es utilizada por las empresas para dar a conocer a nivel corporativo y comercial los productos que ofrece, cuyo resultado es la creación de una marca única para todos sus productos en diferentes líneas (Llopis, 2016). El modelo endosado se basa en la combinación entre la marca corporativa y del producto o empresa, a través de la comunicación y su apoyo total, (Baños \& Rodríguez, 2016). Las marcas independientes se caracterizan ya que cada marca y submarca tiene diferente identidad, es decir, se muestran independientes de la marca de la empresa. En cuanto el modelo híbrido es la combinación de los modelos mencionados combinados según la necesidad de la empresa (León, 2014).

La lealtad de marca va direccionada a la fidelidad de un cliente hacia una empresa, siendo la primera opción en un determinado tipo de producto, esto se logra con la satisfacción del cliente en cada visita que realiza al mejorar su experiencia logrando de esta manera ser un activo más de la organización. (Baños \& Rodríguez, 2016). Para complementar (Rodríguez, 2011) menciona que la lealtad de marca está enfocada a la preferencia de la 
elección de compra hasta llegar a ser una prioridad. Existen diferentes niveles que se pueden identificar lealtad a través de su recuerdo (cuando menciona un producto y lo primero que relaciona el cliente con una marca) y reconocimiento de marca (significa la familiaridad de experiencias positivas de un contacto anterior con un producto)

La conciencia de marca se da a notar en un consumidor reconociendo y recordando un producto o servicio de tal manera que lo asocie a una marca, este proceso debe darse con anticipación antes que su arribo al punto de venta (Curubeto, 2007). El Top of mind es lo primero en la mente del consumidor, ocurre cuando las marcas estas impregnadas en nuestro cerebro, a través de una sencilla pregunta siendo el resultado la marca con mayor índice de compra (Rodríguez, 2011, pág. 235). La notoriedad espontánea mide el realce que tiene una marca, asociándolo rápidamente a un producto, que repercutirá la decisión de compra. La notoriedad asistida hace referencia a la influencia en el proceso de compra por parte de otra persona, al mencionar una marca o recomendarla por su experiencia (Ibidem, Pág. 236).

La calidad percibida es como capta un consumidor un producto por sus atributos físicos ya sean materiales del producto, empaque o imagen de la empresa en el mercado, relación costo beneficio ya que influyen en la adquisición de un producto (Baños \& Rodríguez, 2016). La calidad percibida es la razón por lo que el cliente regresa a una empresa, además es una gran estrategia de diferenciación y posicionamiento permite fijar un precio superior y de esta manera lograr una expansión de la marca (Sainz, 2007).

Las asociaciones de marca están relacionadas hacia la propia marca, que desempeñan un papel fundamental a favor o en contra, es decir, mediante recuerdos que influyen en la decisión de compra, a partir de una experiencia el cliente puede quedar satisfecho o no (Baños \& Rodríguez, 2016). Las asociaciones de marca están relacionadas a la elección de compra ya que al ser evaluada favorablemente tendrá una orientación hacia un producto (Batey, 2013), por lo que se divide en: (1) asociaciones duras (diseño, sabor, textura, estilo, etiqueta y empaque); (2) asociaciones suaves (atributos intangibles o emocionales de un producto).

En el año de 1989 se crea un modelo denominado mapa perceptual que permite a las organizaciones medir su posicionamiento a través de un análisis de una misma categoría, cuyo fin es determinar las marcas competitivas, atributos destacados de los productos, es decir, en qué situación se encuentra la empresa en comparación con la competencia mediante la mente del consumidor de manera gráfica (Jiménez \& Calderón, 2007, pág. 96). Este modelo se compone de: (1) eje horizontal; (2) eje vertical. 
La personalidad de marca se enfoca a los clientes ya que influye fuertemente en el posicionamiento en relación con la competencia, se crea mediante la interacción hacia empleados y clientes. Cabe mencionar que se orienta a la proyección de una imagen de: (1) sinceridad, demostrando que la marca comunica ser honesta; (2) emoción, la marca muestra ser atrevida y actualizada; (3) competencia, establece rasgos identificativos como ser confiable, inteligente y exitosa (Kapferer, 2008 \& De Chematony, 2001).

El territorio de marca hace referencia a la diversificación de los productos de la empresa con la finalidad de aprovechar la imagen de la marca mediante su concepción en la mente del consumidor en el mercado, ayudando a la aceptación de nuevos productos. Por otro lado, la extensión de marca inapropiada desembocaría en el prestigio. El territorio de marca se compone: (1) Nicho - posición; (2) Espacio dimensional. (Costa, 2010; Chailan et al. 2003).

El nicho - posición se enfoca a la expansión de imagen de la marca, se establece un territorio de marca que permite su explotación. La extensión de línea toma como referencia la marca madre hacia un nuevo producto con el fin abarcar nuevo mercado, desembocando en la captación de nuevos clientes. (Keller, 2008; García, 2005). La extensión de categoría se orienta a la aplicación de la marca corporativa teniendo como beneficio a una nueva categoría de productos mediante el posicionamiento que tiene la marca en el mercado. La diversificación es la cantidad de variedad de productos existentes en una empresa, de igual forma se toma en consideración a las submarcas (Costa, 2010; Keller, 2008).

El espacio dimensional es la percepción del consumidor hacia mercado, los directivos toman en consideración la totalidad de productos existentes con el fin de no perder mercado. La totalización de productos se clasifica en: primero en tipos y variedades de productos, en segundo lugar, se encuentran las marcas y submarcas según las líneas de productos, finalmente, la combinación con las anteriormente mencionadas dando como resultado una gran cantidad de productos.

El Dimensionamiento de la superficie surge a partir de las estrategias de extensión de marca, mediante el lanzamiento de nuevas líneas de productos para abarcar mercado a partir de una misma categoría con el fin de lograr el crecimiento de la empresa. (Chailan, Boyer, \& Calderón, 2003; Costa, 2010). 
El agrupamiento de marca es una estrategia que las empresas asumen con el fin de introducirse en el mercado a través del uso de la marca corporativa o submarcas, mediante este agrupamiento permite la planificación de la organización con el fin de atraer la atención del cliente. (Aaker, 2005; Calvo, 2016; Chailan et al. 2003; Keller, 2008). La relación marca - producto hace referencia al vínculo existente con los atributos del producto que lo percibe el consumidor por su diferenciación con la competencia. (Curubeto, 2007). La relación marca - consumidor busca crear una asociación directa con el cliente a través de rasgos distintivos en el concepto de una marca más fuerte para que de esta manera el cliente se identifique con la marca (Keller, 2008; Curubeto, 2007).

La relación marca - empresa se asocia a la cultura empresarial con la sociedad creando conciencia en el consumidor, mediante el cuidado de las personas, medioambiente y tecnológico. En este tipo de relación prevalecen los atributos de la organización con el fin de lograr la identificación del cliente por la contribución de la empresa (Keller, 2008; Curubeto, 2007). Finalmente, la relación marca - marca se da por el reconocimiento de la marca a través de símbolos de manera creativa, ya que al asociar a un logotipo no tendrá el mismo grado de relación mediante la identificación gráfica de un producto el consumidor se siente atraído por una marca logrando posicionarla en el mercado de manera única (Curubeto, 2007; Costa, 2010).

La definición y dimensión del portafolio de marcas admite que la organización gestione eficientemente las marcas a través de la diferenciación de variables dentro de una organización mediante orientaciones de marketing. Cabe mencionar que permite determinar el número de marcas y sus tipos. La definición y dimensión del portafolio de marca abarca lo siguiente: (1) Tipo de marca; (2) Diseño de la estructura de portafolio (Chailan et al. 2003; Morgan \& Rego, 2009)

La tipificación de la marca faculta a la empresa lograr la diferenciación de un producto a partir de su identidad, según la necesidad de cada organización se implantan marcas para cada producto. Cabe mencionar que el tipo de marca dependerá de la relación de marcas (García, 2005). Mediante este contexto el autor clasifica las marcas de la siguiente manera: (1) portafolio; (2) roles; (3) definen el rol del producto.

Las marcas para portafolio proporcionan la evolución histórica de la empresa, los productos que comercializaba. Adicionalmente se considera el vínculo entre las marcas y submarcas (Calvo, 2016). La marca única se la conoce como marca paraguas cuyo fin es diversificar los productos que ofrece una empresa u organización (García, 2005; Cerviño, 2002). 
La marca de división es utilizada por unidades de negocio están orientadas a distintos mercados objetivos mediante varias líneas de productos que cubre la marca principal (Cerviño, 2002). La marca mixta es manejada para el aprovechamiento de la marca principal, además de aportar dos marcas al producto. La marca individual desarrolla su identidad en el mercado a través de marketing satisfaciendo necesidades del mercado meta (Keller, 2008; García, 2005; Cerviño, 2002)

Las marcas con roles definen el papel del producto a través de la orientación al consumidor, la cual posee la siguiente categorización: (1) marcas madre; (2) marcas de respaldo aportando firmeza en la oferta; (3) submarcas con orientación producto - mercado; (4) descripción de ofertas, orientando a los consumidores a conocer la funcionalidad de un producto; (5) definir marcas y submarcas según su línea de productos; (6) marca paraguas, aprovechamiento del prestigio de la marca principal; (7) marcas modificadoras que se diferencian según su calidad y atributos; (8) marcas relacionadas de diferentes organizaciones originando grandes ofertas para el cliente; (9) roles conductores que reflejan la decisión de compra (Aaker, 2005; Keller, 2008).

El diseño de la estructura de portafolio constituye un indicador de gestión óptimo de marca, hay que tomar en consideración elementos de costos y rentabilidad. Se diseñan a través de los territorios de marcas y su potencial (Chailan et al. 2003), teniendo como resultado la apreciación por parte del cliente a un determinado producto que se lo realiza de la siguiente manera: (1) pilas de marca, coordinación entre marca global y submarcas para identificar la variedad de productos; (2) parque de marcas, manejo combinado entre marca paraguas y categorización de marcas dando a conocer distintos tipos de productos; (3) torre de marcas, tomando como referencia la reputación de la empresa y experiencia del cliente en cada producto; (4) silos de marca, valor añadido al producto mediante estrategias aplicadas según el tipo de producto y su segmento (Muylle, Dawar, \& Rangarajan, 2012).

La gráfica de la cartera de marca permite la creación de una estructura de identidad a través de elementos que componen un concepto. Estos elementos son integrados por la forma verbal y visual (isotipo, logotipo, imagotipo) cuyo propósito es realizar un análisis de identidad, protección legal de marca (Cerviño, 2002; Costa, 2010). Comenzando con el concepto del producto o servicio donde se detalla la percepción del cliente hacia una organización que interviene: (1) proposición, la promesa de marca a través de ofertas; (2) personal, expectativas percibidas por el cliente; (3) procesos, forjar valor para los consumidores; (4) productos, evaluación de diferenciación de un producto en el mercado (Clifton, y otros, 2003). 
El nombre de la marca es la identificación verbal en el mercado que capta la esencia de la empresa cuya finalidad es posicionarse en la mente del consumidor para lo cual debe considerar lo siguiente: (1) nombre de la marca debe ser simple, además de tener una fácil pronunciación y escritura; (2) debe familiar, es decir, objetos cotidianos para el cliente y significativo para que sea de fácil recordación; (3) ser único y distintivo (Keller, 2008).

Hace referencia a los atributos tangibles del producto como son diseño y envase, es decir, la manera de cómo será el resultado final del producto se anunciará al cliente. Adicionalmente la presentación del producto es la envoltura que posea el producto no solo sirve para protégelo sino se lo realiza de manera creativa - llamativa (Keller, 2008).

El concepto creativo de comunicación y comercialización son elementos de difusión interna y externa. La comunicación interna se da a partir de papelería, identificación del personal, etc. La comunicación externa se lo realiza en medios de comunicación, señalética, patrocinio. Comprende las actividades de la empresa con el fin de lograr su reconocimiento en el mercado (García, 2005).

El registro y protección jurídica confiere el uso exclusivo de una marca a una empresa o su representante legal. Hay que tomar en consideración que se registra tanto el nombre de la empresa como su logotipo y colores, eslogan, colores corporativos con el fin de evitar que empresas con productos similares se beneficien del prestigio de la marca (Cerviño, 2002).

En la última etapa se valora cada uno de los elementos que intervienen en la construcción de una marca. El logotipo es la representación escrita y gráfica de una marca a través de su nombre, añadiendo atributos institucionales que generan distintivo en la marca siendo visibles e identificables. El símbolo son formas o gráficos que identifican visualmente ya sea una empresa o una marca. La identidad cromática hace referencia a los colores representativos de la marca mediante la relación de color con lo que representa psicológicamente para el consumidor. La tipografía representará un producto o empresa, la denotación de cada tipo de letra es identificativa para el cliente.

Finalmente, el nombre de la marca hace referencia a la representación lingüística de la marca, (Costa, 2010 \& Llopis 2011). Dentro del contexto posicionamiento, el perfil de percepción de marca se establece mediante asociaciones percibidas logrando la memorización de la marca de elementos representativos de la empresa, (Vera, 2008). El top 
of mind, hace referencia a la primera respuesta que mencione el cliente podemos conocer cuál marca impregnada en la mente del consumidor en primer lugar. (Hall, 2017).

La propuesta única de ventas permite a la organización diferenciarse del mercado, primero enfocarse al público objetivo, de manera que a largo plazo permitirá ampliar el mercado objetivo, el segundo lugar se debe aprovechar las dificultades de los competidores, luego al ofrecer un producto al mercado que sea único, finalmente, conocer los gustos y preferencias de los clientes permitirá descubrir su comportamiento (Osterwalder, Pigneur, Smith, \& Bernarda, 2015).

\section{Marco metodológico.}

El proyecto se basa en el constructivismo a partir de la interacción entre el investigador y el grupo objetivo de estudio. El autor se relacionó con los propietarios de Pymes de calzado y por otro lado consumidores del producto lo que permitió el enriquecimiento del conocimiento (Ramos, 2015).

La investigación fue realizada en la provincia de Tungurahua República del Ecuador en el mes de enero del 2018. Para la recolección de información se utilizó dos instrumentos. Para esto, se capacitó a 26 individuos los cuales ayudaron a la obtención de información, de los cuales 16 aplicaron una encuesta estructurada a consumidores de calzado de la provincia y los restantes 10 realizaron una entrevista formal con los propietarios o encargados de empresas de Pymes de la provincia.

En la caracterización teórica se adoptó por el método histórico lógico, de esta manera, se ubicó el objeto de estudio en el tiempo, con el fin de explicar su origen y evolución.

El método inductivo - deductivo, permitió direccionar a la investigación a través del estudio de la teoría general del branding, obteniendo antecedentes acerca de la arquitectura, identidad y posicionamiento. Para el método analítico sintético se analizaron distintos modelos tanto de arquitectura, identidad y posicionamiento de la marca. Finalmente, el método hipotético hace referencia a la pregunta de investigación que a partir de un modelo teórico repercutirá en el posicionamiento en las empresas de calzado.

Para la validación del modelo teórico fue mediante el programa Smart PLS (Partial Least Squares), a partir de mínimos cuadrados parciales, en el gráfico 1 se muestra la relación que existente entre la gestión de marca y sus indicadores teniendo como lo principales al territorio de marca $(0,906)$, gráfica de cartera $(0,925)$ y agrupamiento de marca $(0,708)$. 
Gráfico 1: Resultado del modelo de ecuaciones estructurales de arquitectura, identidad y posicionamiento de marca en Pymes del sector de calzado de Tungurahua.

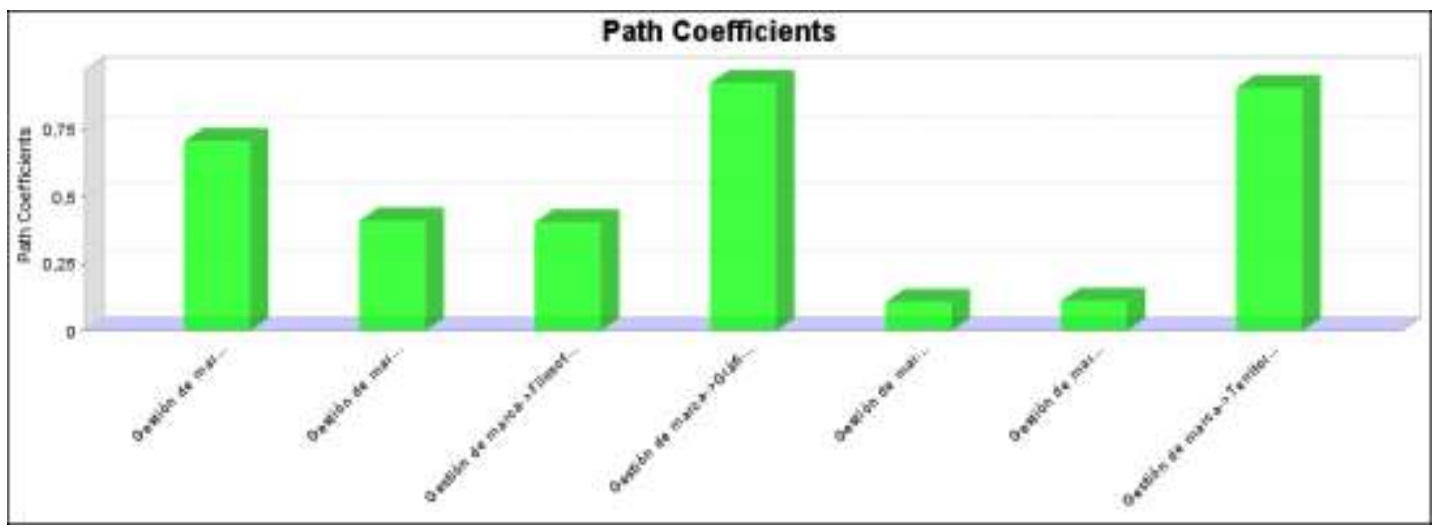

Fuente: Smart PLS.

Elaborado por: Los autores.

En la figura 1, se aprecia el peso y magnitud a través de las conexiones entre los indicadores del constructo. Comenzando con los coeficientes de determinación $\mathrm{R}^{2}$ (círculos), tenemos que el territorio de marca $(0,821)$ y la gráfica de cartera $(0,855)$ tienen un alto poder explicativo, mientras que el agrupamiento de marca $(0,501)$ posee un nivel medio de poder explicativo. Al examinar los vínculos de causalidad de los indicadores independientes y dependientes.

Figura 1: Vínculos del constructo de arquitectura, identidad y posicionamiento de marca a través de mínimos cuadrados parciales.

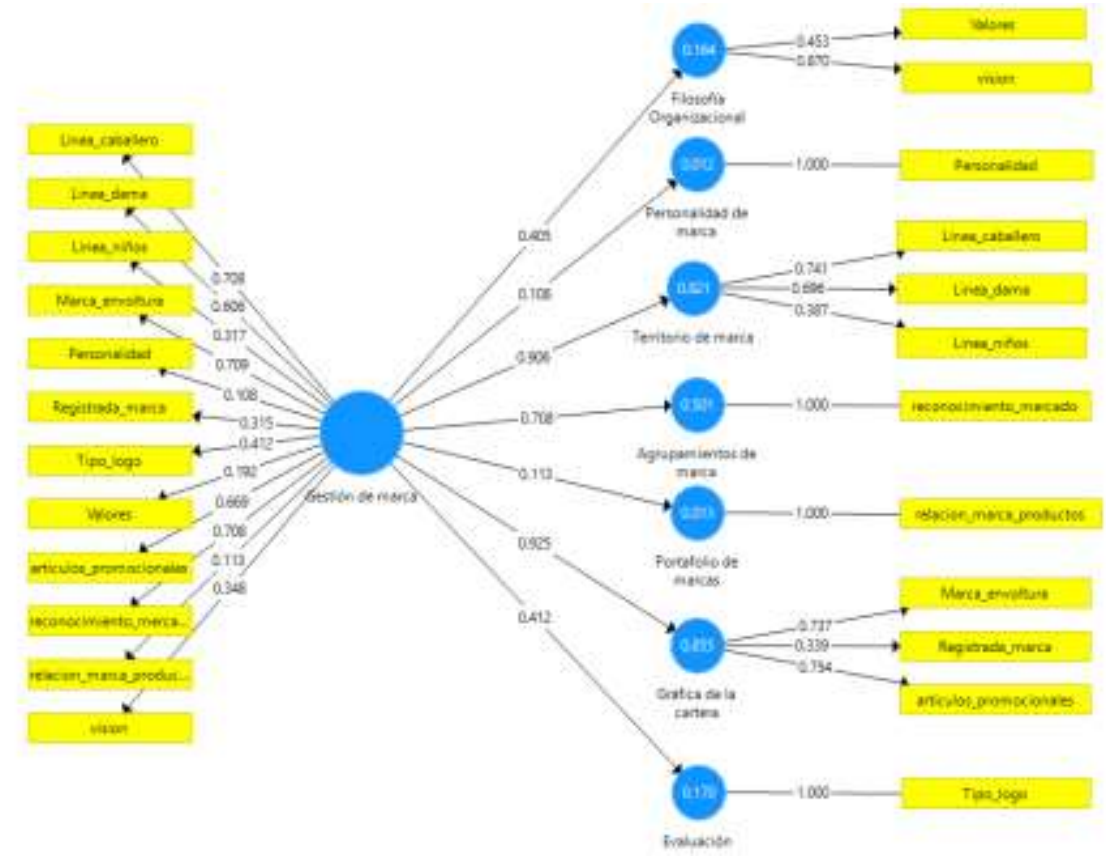


Elaborado por: Los autores

Los métodos empíricos utilizados en la investigación fueron la construcción de un sistema categorial a través de la operalización de variables lo que desemboco en cuestionarios estructurados. Para la recopilación de datos, fue a través de la técnica de entrevista a profundidad estructurada que se dividía en datos de la empresa, información de la identidad de la empresa, información del producto e información de la identidad de la marca para determinar cómo fue la creación de marca y como gestiona en las empresas.

En segundo lugar, la técnica de encuesta estructurada que consta de 10 preguntas para conocer el posicionamiento de las marcas nacionales a partir del criterio del consumidor de calzado.

Existen dos poblaciones de estudio, la primera engloba a las pymes de la provincia de Tungurahua, que según (Villavicencio, 2013) el 50\% de la industria nacional de Pymes corresponden a Tungurahua siendo 200 empresas de las cuales se tuvo aceptación de 100 empresas, es decir, dieron apertura para obtención de datos. La segunda es la población económicamente activa de Tungurahua siendo 244.893 habitantes, teniendo como muestra a 384 habitantes consumidores de calzado. Posteriormente se depuró los datos para verificar si se encontraban debidamente llenadas para luego tabularlas en el programa estadístico SPSS para generar gráficos estadísticos de frecuencias para luego interpretarlos.

\section{Análisis e interpretación de resultados.}

Resultados de la entrevista aplicada a 100 gerentes de pymes de la provincia, gráfico 2 acerca de la representación de la marca teniendo con mayor participación la imagen asociándolo con la competitividad con el 59\%, el $22 \%$ de los gerentes mencionaron que su imagen es sincera, el $14 \%$ de los entrevistados relacionan la imagen de su marca es emocional y el restante 5\% lo relaciona a la rudeza. En cuanto a la estructura o composición de la marca, tabla 1, el $66 \%$ de los propietarios de empresas de pymes muestran composición de logotipo en sus marcas, mientras que el 17\% tiene una marca con imagotipo, el $11 \%$ de los entrevistados mencionan que poseen isotipos en su marca y el restante $6 \%$ creo su marca a partir de un isologo.

La relación que acopla la marca con los productos según la gestión de los gerentes, como se muestra en el gráfico 3: se lo maneja con el 71\% la marca es única y por ende es imagen de todos sus productos, mientras que el $20 \%$ menciona que posee una marca para cada línea producto y el $9 \%$ restante utiliza una marca para cada tipo de producto. A partir de la encuesta aplicada a los consumidores de calzado con el preámbulo de 4 marcas mencionadas por el consumidor, en gráfico 4 el $42 \%$ de los encuestados mencionan que las 
marcas de calzado nacionales la relacionan con estilo, el $31 \%$ asocia las marcas con el confort que presentan sus productos, la asociación de las marcas nacionales con el estatus representa el $15,65 \%$ y el restante $10 \%$ asocia las marcas con el color.

El top 10 de empresas nacionales reconocidas por la mente del consumidor de calzado, a través de la encuesta se pidió a los encuestados mencionar 4 empresas de calzado nacional teniendo como los más representativos en la tabla 2: Venus con el 22,9\%, Gamos 20,1\%, Bunky,16,8\%, Lady Rose 9,5\% entre los más recordados.

Tabla 1. Top 10 empresas nacionales de calzado.

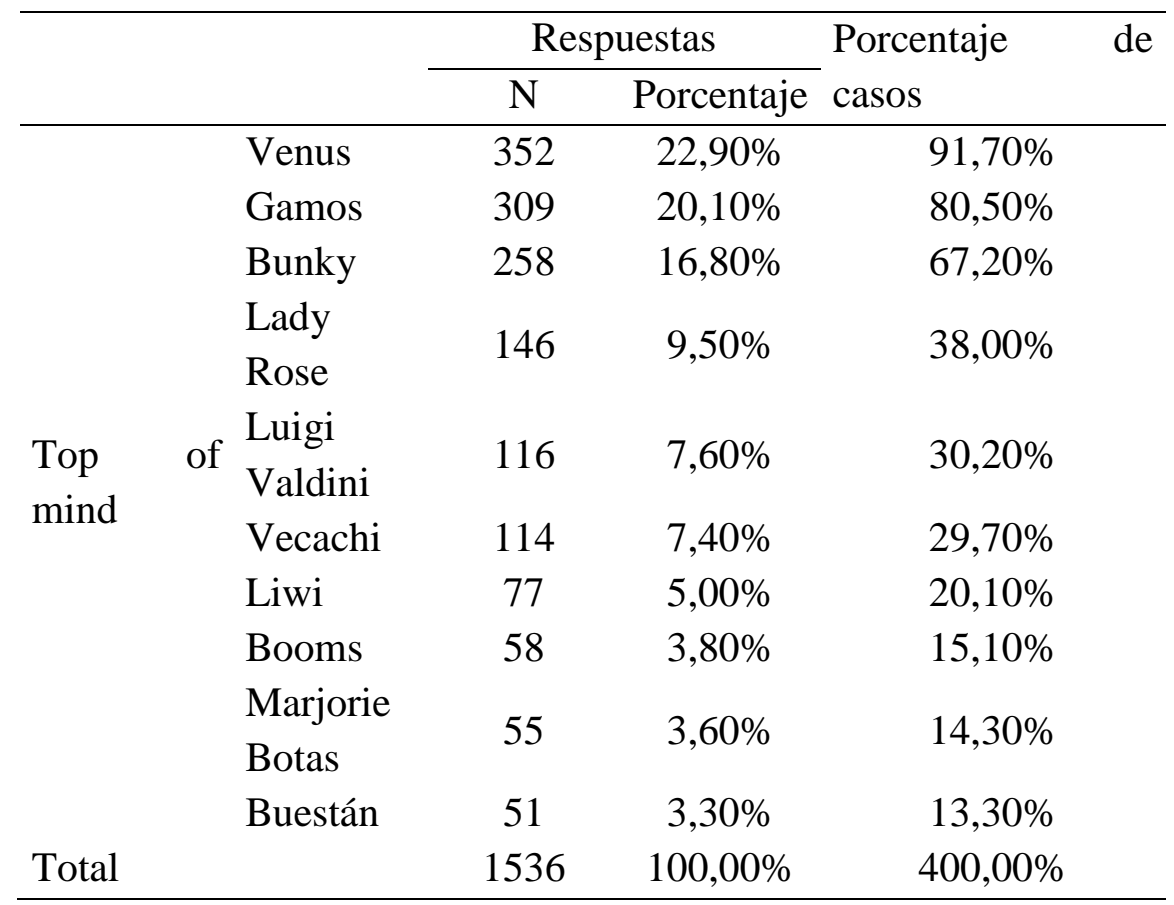

Fuente: Los autores, 2018.

El nivel satisfacción del consumidor de calzado a través de su experiencia de uso menciona que en el gráfico 5, que se encuentran satisfechos $(67,19 \%)$ con el calzado de la provincia, el $19,53 \%$ se encuentra muy satisfecho con su producto, mientras que el 10,68\% es indiferente su nivel de uso con el calzado. A través del programa SPSS según los datos obtenidos en la encuesta sobre las 4 marcas más recordadas y el nivel de satisfacción del consumidor, se ejecutó un análisis de regresión logística, se muestra el 86,2\% de probabilidad de acierto en el resultado de la variable dependiente, es decir, el nivel de satisfacción es satisfactorio y muy satisfactorio. 
A través de la prueba de Hosmer y Lemeshow tabla 6, indica que la varianza explicada por el modelo explica el 82,4\% de la varianza de la variable dependiente (Chi cuadrado 2,177; p .824), Para el análisis de regresión logística en bloque 1 indica que hay un 86,2\% de probabilidad de acerito en el resultado de la variable dependiente, cuando conozco el nivel de satisfacción de los consumidores. Si (ExpB) valor mostrado en la tabla 2 es menor a 1 , aumenta el valor de las variables independientes y disminuye el de la dependiente como el caso de Venus, Gamos. Por otro lado, si es mayor a 1 aumenta el valor de las variables independientes y aumentan el de la dependiente (Bunky, Lady Rose). La puntuación de Wald para el modelo probado indica que las variables independientes aportan significativamente a la predicción de la variable dependiente, este resultado se puede generalizar a la población.

Tabla 2. Variables en la ecuación.

\begin{tabular}{llllllll}
\hline & & B & $\begin{array}{l}\text { Error } \\
\text { estándar }\end{array}$ & Wald & gl & Sig. & Exp(B) \\
\hline & Venus(1) & $-1,067$ & 0,353 & 9,12 & 1 & 0,003 & 0,344 \\
Paso 1 $^{\text {a }}$ & Gamos(1) & $-0,062$ & 0,456 & 0,018 & 1 & 0,892 & 0,94 \\
& Bunky(1) & 1,418 & 0,506 & 7,842 & 1 & 0,005 & 4,13 \\
& Lady_Rose(1) & 0,269 & 0,309 & 0,758 & 1 & 0,384 & 1,309 \\
& Constante & 1,893 & 0,298 & 40,425 & 1 & 0 & 6,637 \\
\hline
\end{tabular}

a. Variables especificadas en el paso 1: Venus, Gamos, Bunky, Lady_Rose.

Fuente: Los autores, 2018.

Para calcular la invarianza del modelo teórico se aplicó el programa SPSS módulo Amos donde se tomó en consideración las variables ordinales del modelo como son: personalidad; tipo de logo; relación marca productos; valores. A partir del chi cuadrado y df obtenidos del programa SPSS módulo Amos, calcular la invarianza a través de "Start Tools Package", teniendo como resultado la existencia de una invarianza

Figura 2: Relación variables programas SPSS módulo Amos. 


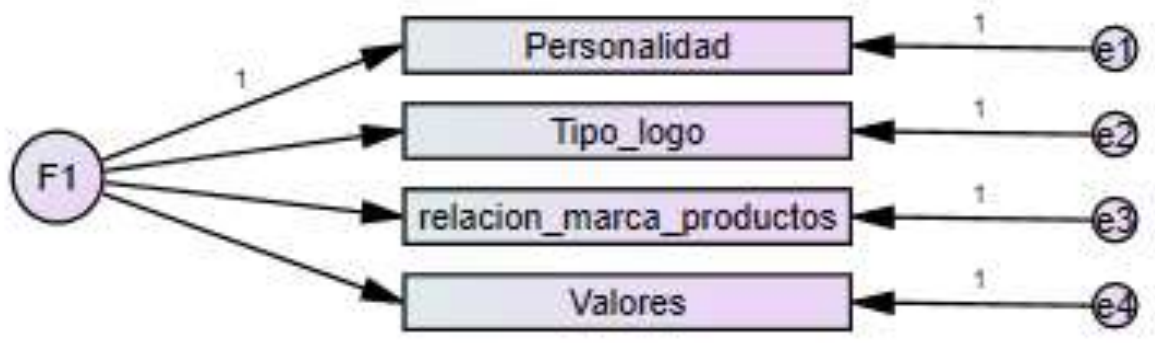

Fuente: Los autores, 2018.

\section{Conclusiones.}

- Los rasgos o cualidades que presentan las empresas de la provincia dedicados a la elaboración de calzado son competitivos, siempre buscan sobresalir de las demás empresas y la mejor forma de demostrarlo es a través de la proyección de su imagen (marca), la personalidad sincera trasmite honestidad en su labor que desempeñan que va de la mano con la personalidad emocional que se apodera de una situación social creando un vínculo directo con el consumidor. Por otro lado, la personalidad ruda hace que los consumidores sientan valentía.

- El tipo de marca es utilizado por las empresas de calzado, muestran el nivel de complejidad de la aplicación de la marca de tal manera que el logotipo al ser un elemento simple identificado por un texto tiene mayor presencia, en cuanto al imago tipo pocas empresas han desarrollado sus marcas bajo este paradigma por la combinación entre imagen y texto que funcionan por separado. El isotipo es representado con menor número de empresas que lo posee ya que buscan una sola imagen o figura, es decir, simbólicamente sin un texto, mientras que en el mismo grado de importancia el isologo muestra la unión de texto e imagen que funciona únicamente juntos por su nivel de dificultad no es acogido claramente por las empresas del sector.

- Los directivos de las empresas de calzado gestionan su marca de tal manera que en su mayoría muestre la misma imagen de la marca en todos sus productos con el fin de posicionarse en la mente del consumidor, mientras que también existen empresas que implantan a cada línea de sus productos una marca para diferenciarse y que el consumidor sienta atracción, Por otro lado, pocas empresas adoptan el crear una marca para cada tipo de producto porque es demasiado costoso por la inmersión de la marca en el empaque, dependiendo del tamaño de la organización puede ser posible.

- Los consumidores de calzado asocian sus marcas favoritas al estilo, con la atracción mediante el diseño de sus productos llegando al favoritismo. También en la provincia relacionan las marcas con el confort por su experiencia de uso y la 
recomendación por parte de terceros. La relación por estatus es baja ya que existen pocas marcas que proyecten un gran nivel de estatus por lo que es accesible para pocos consumidores. Finalmente, muy pocos consumidores de calzado recuerdan una marca por sus colores representativos debido a la construcción y posicionamiento de su marca.

- Las empresas más representativas del sector de calzado en la nación son el resultado de una correcta gestión de marca debido al posicionamiento en la mente del consumidor, primero por su marca al tener una correcta construcción y aplicación de marca tal es el caso de Venus que está impregnado en la mente del consumidor ecuatoriano, la simplicidad de su nombre que al igual de su isotipo o trazo es de fácil recordación para el consumidor como es el caso de Bunky; Vecachi; Lady Rose; Buestán; Booms. Segundo por el prestigio de calidad de sus productos como son: Gamos; Luigi Valdini; Liwi; Marjorie Botas.

- El nivel de satisfacción que muestra el consumidor Tungurahuense es satisfactorio por su experiencia de uso a partir de la calidad del producto, materia prima y sobre todo la durabilidad del producto. Al no ser muy satisfactorio su nivel de satisfacción se debe a pequeños detalles que vinculan a un cliente con la marca, un servicio postventa, etc. Por otro lado, es mínimo el nivel de insatisfacción de consumidor debido atributos que presenta el producto.

\section{Referencias bibliográficas.}

Aaker, D. (2005). Estrategia de la cartera de marca: crear relevancia, difeenciación, energía, apalancamiento y claridad. España: Gestión 2000.

Águeda, E. (2008). Principios de marketing. ESIC Editorial.

Baños, M., \& Rodríguez, T. (2016). Imagen de marca y product placement. ESIC.

Batey, M. (2013). Significado de la marca: Como y por que ponemos sentido a productos y servicios. Ediciones Granica.

Belío, J. (2007). Claves para gestionar precio producto y marca: cómo aforntar una guerra de precios. Especial Directivos.

BID, B. D., \& INTAL, I. (2000). El impacto sectorial del proceso de integración subregional en el MERCOSUR: sector calzado y sector farmacéutico. Serie RED Int.

Calvo, J. (2016). Marca Holística de moda. Madrid, España: Dykinson Editorial. 
Campoy, D. (2007). Cómo gestionar y planificar un proyecto en la empresa: Técnicas y métodos para el éxito de un proyecto empresarial. España: Ideas propias.

Capriotti, P. (2009). Branding Corporativo: Fundamentos para la gestión estratégica de la identidad corporativa. Santiago de Chile.

Cedolin, L., \& Angeles, S. (2015). El Brading made in Spain: la marca España en la internacionalización de las marcas de calzado y moda. Universitat Ramon Llull.

Cerviño, J. (2002). Marcas Internacionales Como crearlas y gestionarlas. Madrid: Ediciones Pirámide (Grupo Anaya, S.A.).

Chailan, C., Boyer, A., \& Calderón, L. (2003). Portafolio de marcas: Un marco conceptual. esan-cuadernos de difusión.

Clifton, R., Simmons, J., Ahmad, S., Allen, T., Anholt, S., \& Bahr, A. (2003). The Economist Brands and Branding. London: Profile Books Ltd.

Collins, J., \& Jenrry, P. (1996). Building your company's visión. Harvard Business Review.

Comercio, G. E. (21 de Enero de 2016). Obtenido Bestán y Kleiner ayudarán a sustituir USD 60 millones en importaciones de botas industriales. El Comercio. Obtenido de http://www.elcomercio.com/actualidad/buestan-kleinerfabricacionbotasindustriales.html

Costa, J. (2010). La marca: creación, diseño y gestión. México: Trillas.

Curubeto, C. (2007). La marca universitaria: atributos, beneficios o valores: ¿Qué utlizan las universidades de Buenos Aires para construir su identidad de marca y su propuesta de valor? Universidad Austral.

Davis, S. (2002). La marca: Máximo valor de su empresa. México: Pearson Education.

De Chernatony, L. (2001). A model brand for strategically building brands. Brand Management.

Díaz, L. (2017). Soy marca: Quiero trabajar con influencers. Profit Editorial.

Erreguerena, M. (2013). La construcción de la memoria coletiva sobre los hechos democratizantes: Estados Unidos en la sesenta y la serie de televisión Mad Men.

ESIC. (2015). 50 años de marketing. ESIC Editorial.

Ezenarro, J. (2014). Elementos de identidad de marca vs elementos lovemarks. Universidad Católiica Andres Bello. 
Gámez, R., Soria, R., \& López, C. (2006). Organizaciones y políticas públicas: una mirada desde el noroeste. México.

García, M. (2005). Arquitectura de marcas: modelo general de construcción de marca y gestión de sus activos. Madrid, España: ESIC Editorial.

Guajala, M., Mantilla, L., Mayorga, C., \& Moyolema, M. (diciembre de 2015). Procesos de producción y productividad en la industria de calzado ecuatoriana: Caso empresa Mabelyz. Revista ECA Sinergía(7), 88-100.

Hall, J. (2017). Top of Mind: Use content to unleash your influence and engage those who matter to you. McGraw - Hill.

Hamish, W. (2007). Em Sintonia con a Marca. Editora Cultrix.

Hatch, M., \& Schultz, M. (2010). Ensencia de marca. LID Editorial.

Hoyos, R. (2016). Branding el arte de marcar corazones. Eoce Ediciones.

Jácome, H., Naranjo, M., \& Mayo. (2010). Boletín mensual de análisis sectorial de MIPYMEs Sector Calzado. Centro de Investigaciones Económicas y de la Micro Pequeña y Mediana Empresa, y Ministerio de Industrias y Productividad.

Jaramillo, P., Riviera, A., \& Ponce, L. (2011). La marca en la configuración y funcionamiento de la empresa: origen, evolución y relevancia.

Jiménez, A., \& Calderón, H. (2007). Dirección de productos y marcas. UOC Editorial.

Jurado, J. (2016). Cómo convertir TU EMPRESA en un MARCA LÍDER en el Mundo. Mestas Ediciones.

Kapferer, J. N. (2008). The new strategic brand management: Creating and sustraining brand equity long term. London: Kogan page.

Keller, K. (2008). Adminsitración estratégica de marca. México: Pearson Education.

King, K., \& Varela, M. (2012). Boletín mensual de análisis sectorial de MIPYMES Estudio de caso sector cuero y calzado: Calzado Gamos. Centro de Investigaciones Económicas y de la micro pequeña y mediana empresa, Ministerio de Industrias y Productividad.

Kissel, P., \& Büttgen, M. (2015). Using social media to communicate employer brand indentity: The impact on corporate image and employer attractiveness. Journal of Brand Management.

Kotler , P., \& Keller, K. (2012). Dirección de Marketing. México: Pearson Education. 
Kotler, P. (2009). Dirección de Marketing. Pearson Educación.

Kotler, P., \& Armstrong, G. (2012). Marketing. México: Pearson Education.

Kotler, P., \& Kotler, M. (2014). 8 maneras de crecer: estrategias de marketing para desarrollar tu negocio. LID Editorial.

Krupatini, S. (2011). Y ahora qué hacemos ante la complejidad un abordaje teórico práctico para la gestión de empresas y gobiernos en entornos turbulentos. Granica.

León, J. (2014). Change Marketers: La empresa como agente de cambio. EL Viso Media S. L. U.

Llopis, E. (2016). Crear la marca global modelo práctico de cración e internacionalización de marcas. ESIC Editorial.

Morgan, N., \& Rego, L. (2009). Brand Portfolio Strategy and Firm Performance. Journal of marketing. American Marketing Asociation, 1(73), 59-74.

Muylle, S., Dawar, N., \& Rangarajan, D. (2012). B2B Brand Architecture. Cmrberkeley edu.

Ocaña, H. R. (2012). Dirección estratégica de los negocios. Buenos Aires: Dunken.

Olins, W. (2009). El libro de la smarcas. London: Océano Editorial.

Olmedo, R. (2010). El valor de la marca en época de crisis. Argentina: Universidad de Palermo.

Osterwalder, A., Pigneur, Y., Smith, A., \& Bernarda, G. (2015). Diseñando la propuesta de valor: como crear los productos y servicios que tus clientes están esperando. España: Grupo Planeta Spain.

Ramos, C. (2015). Los paradigmas de la investigación cinetífica. Scientific research paradigms.

Rodríguez, I. (2011). Principios y estrategias de marketing: incluye web. Editorial UOC .

Romo, D. (2015). Análisis de las estrategias de Branding emocional y sensorial utilizadas por starbucks en Estados Unidos y prpuesta de un modelo para su aplicación al negocio de cafeterias en el norte de la ciudad de Quito. Ecuador: PUCE.

Rosser, C. (2007). Cómo vender a clientes resistentes (Segunda ed.). FC Editorial.

Sainz, J. (2007). La distribución comercial: opciones estratégicas. ESIC Editorial. 
Stanton, W., Etzel, M., \& Walker, B. (2007). Fundamentos de Marketing. México: McGraw-Hill.

Vaca, G. (2011). La construcción de una marca existosa y estrategias de Branding para manejar las marcas Ecuatorianas. Ecuador: UDLA.

Vega, T. (2007). Aplicación del Branding en CARE Ecuador. UDLA.

Vela, J. (2012). Teoría y métodos para marcas de territorio. Editorial UOC.

Velilla, J. (2010). Branding: Tendencias y retos en la comunicación de marca. España: Editorial UOC.

Vera, J. (2008). Perfil de valor de marca y la medición de sus componentes. Bogotá: Academia revista latinoamericana de administración.

Vicente, J. (2008). e-Branding: posiciona tu marca en la red. Netbiblo.

Villaseca, D. (2014). Innovación y marketing de servicios en la era digital. ESIC Editorial.

Villavicencio, L. (2013). PYMES como eje central de la cadena productiva en el Ecuador.

Villegas, D., \& Zapata, H. (2007). Competitividad sectorial interncional Caso: sector del cuero y del calzado. Entramado.

Whitehill, A. (1994). La gestión empresarial japonesa: tradición y transición. (A. Bello, Ed.) Santiago de Chile. 


\section{Para citar el artículo indexado.}

Suárez L., Saltos J. \& Beltrán C. (2018). Posicionamiento, arquitectura e identidad de marca: un estudio correlacional en el sector calzado de Tungurahua. Revista electrónica Ciencia Digital 2(2), 91-109. Recuperado desde:

http://cienciadigital.org/revistacienciadigital2/index.php/CienciaDigital/article/view/75/70

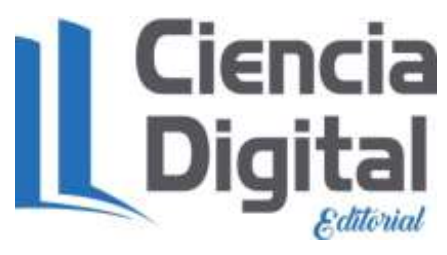

El artículo que se publica es de exclusiva responsabilidad de los autores y no necesariamente reflejan el pensamiento de la Revista Ciencia Digital.

El articulo queda en propiedad de la revista y, por tanto, su publicación parcial y/o total en otro medio tiene que ser autorizado por el director de la Revista Ciencia Digital.
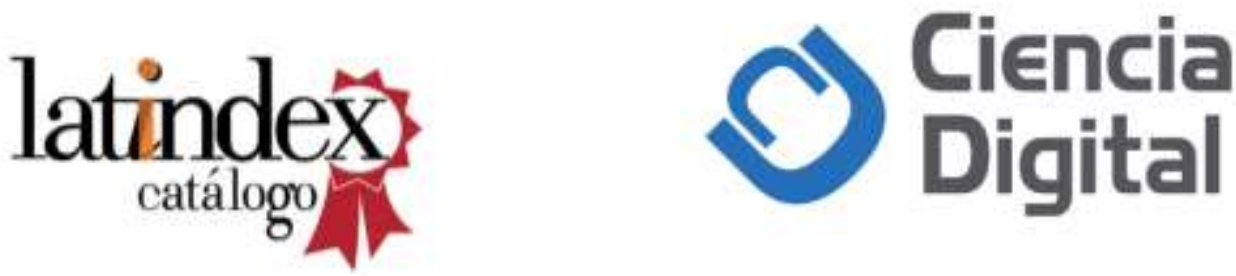